Meta

Journal des traducteurs

Translators' Journal

\title{
Interview de Mme Arlette Morel, présidente de la Fédération nationale des sourds de France
}

\section{Danica Seleskovitch}

Volume 42, numéro 3, septembre 1997

L’interprétation en langues des signes

URI : https://id.erudit.org/iderudit/004082ar

DOI : https://doi.org/10.7202/004082ar

Aller au sommaire du numéro

Éditeur(s)

Les Presses de l'Université de Montréal

ISSN

0026-0452 (imprimé)

1492-1421 (numérique)

Découvrir la revue

Citer ce document

Seleskovitch, D. (1997). Interview de Mme Arlette Morel, présidente de la

Fédération nationale des sourds de France. Meta, 42(3), 560-563.

https://doi.org/10.7202/004082ar
Résumé de l'article

Les sourds ne pouvant être une communauté isolée, ils se doivent d'être bilingues français-LSF. On examine le rôle que l'interprétation peut jouer pour développer leur maîtrise du français et leur donner un accès plus aisé à la culture. 


\title{
INTERVIEW DE MME ARLETTE MOREL, PRÉSIDENTE DE LA FÉDÉRATION NATIONALE DES SOURDS DE FRANCE
}

\author{
DANICA SELESKOVITCH \\ Professeur émérite, Université Paris III-Sorbonne Nouvelle (ESIT), Paris, France
}

\begin{abstract}
Résumé
Les sourds ne pouvant être une communauté isolée, ils se doivent d'être bilingues français-LSF. On examine le rôle que l' interprétation peut jouer pour développer leur maîtrise du français et leur donner un accès plus aisé à la culture.
\end{abstract}

\begin{abstract}
The author explains why the Deaf cannot form a selfcontained community but have to be French-LSF bilinguals, and shows how interpretation can enhance deaf people's mastery of French and facilitate their general cultural advancement.
\end{abstract}

Danica Seleskovitch: Madame la Présidente, chère amie, le fait que les sourds revendiquent la LSF comme moyen d'expression et réclament des interprètes en LSF ne traduitil pas une certaine forme de repli sur soi de la part de la communauté sourde, un rejet de la langue française?

Arlette Morel : En France, la langue des signes a été interdite pendant un siècle dans les classes de jeunes sourds. Si les pratiques pédagogiques ont commencé à évoluer depuis une vingtaine d'années, la loi qui autorise le bilinguisme, c'est-à-dire l'utilisation conjointe du français et de la langue des signes dans les écoles, ne date que de 1992. Les spécialistes de la surdité dans leur majorité considéraient avec beaucoup de mépris la langue des signes, ne lui reconnaissant pas le statut de langue. Grosso modo, on pensait qu'il s'agissait d'un moyen de communication fruste qui n'était qu'un pis-aller et devait être réservé aux moins doués des sourds. Il a fallu venir à bout des préjugés; c'est pourquoi, dans un premier temps, la reconnaissance de la LSF comme langue naturelle des sourds a mobilisé toutes les énergies. Depuis 1975, date à laquelle on peut situer approximativement l'émergence de cette revendication chez les sourds, les choses ont considérablement évolué, et ce, pour plusieurs raisons.

Les sourds entretenaient des relations avec le milieu entendant essentiellement dans le cadre du cercle familial, de l'environnement professionnel et des liens qu'ils pouvaient conserver avec leurs anciens professeurs. Leur lutte pour la reconnaissance de la LSF et le bilinguisme a permis aux sourds d'élargir considérablement le champ de leurs relations avec les entendants. En effet, toutes sortes d'entendants, linguistes, psychologues, sociologues, spécialistes des sciences de l'éducation, artistes, comédiens et danseurs se sont intéressés à partir de ce moment-là à la LSF et aux sourds. En retour, ces derniers sont devenus assidus aux conférences données par les chercheurs qui abordaient la surdité, aux manifestations consacrées à des problèmes de société de portée générale, ils ont cré des troupes de thêâtre et ont participé à des spectacles mêlant sourds et entendants.

Dans le même temps, bon nombre d'établissements spécialisés dans l'enseignement aux sourds ont évolué progressivement en matière d'éducation. Au début de cette évolution,

Meta, XLII, 3, 1997 
il s'est agi non seulement d'introduire la LSF dans le projet pédagogique mais aussi de fournir aux jeunes sourds des modèles d'adultes sourds. En effet, l'immense majorité des enfants sourds ont des parents entendants et, de ce fait, s'ils ne sont pas mis en présence d'adultes sourds comme eux, ils ne peuvent pas se projeter dans l'avenir. On cite souvent le cas de jeunes sourds qui pensaient qu'au terme de leur scolarité ils deviendraient entendants ou bien qu'ils mourraient; tout simplement parce qu'ils n'avaient jamais vu d'adultes sourds et que, dans le cadre d'une pédagogie strictement oraliste, la communication à propos de tels sujets avec de jeunes enfants était difficilement envisageable. Dans un premier temps, les établissements spécialisés ont embauché des sourds pour faire fonction d'éducateurs, animer des ateliers de communication en LSF, aider les enseignants entendants, donner des cours de LSF au personnel. Puis, la présence des sourds dans les établissements s'est en quelque sorte banalisée. Désormais, il ne s'agit plus seulement d'animer des ateliers de communication en LSF, mais de donner un enseignement structuré de la LSF aux élèves, d'assurer en LSF des cours qui relèvent de l'enseignement général. Récemment, les sourds ont eu accès aux diplômes d'éducateur et de professeur de jeunes sourds. Ils sont encore trop peu nombreux et pas assez reconnus en tant que professionnels, mais le fait est significatif. Les sourds joueront dorénavant un rôle de plus en plus actif dans l'éducation des sourds en tant qu'éducateurs, professeurs de LSF ou professeurs d'enseignement général.

De nombreuses associations se sont créées pour donner des cours de langue des signes aux parents d'enfants sourds, aux professionnels de la surdité et à toutes les personnes intéressées. Par le truchement de l'interprétation, les sourds ont eu accès à un éventail de formations autrement plus large que celui proposé par les institutions traditionnelles. À cette occasion, ils ont été accueillis dans des établissements ordinaires.

Les sourds, pour promouvoir la reconnaissance de la LSF et faire entendre leurs besoins en matière d'interprétation, ont été confrontés à tous les rouages de l'administration, ont rencontré leurs députés, les représentants des pouvoirs publics, sont apparus à la télévision; une jeune actrice sourde a obtenu récemment une distinction nationale importante. Il existe maintenant une émission entièrement réalisée en LSF par des sourds.

Enfin, de manière générale, le projet éducatif pour les sourds est plus ambitieux que par le passé. Il y a une vingtaine d'années, les sourds n'accédaient qu'exceptionnellement aux études supérieures; ce n'est plus tout à fait vrai maintenant.

Contrairement à toute attente, en affirmant leur identité et leurs besoins spécifiques, en participant à la prise en charge de la surdité, les sourds ont été amenés à fréquenter beaucoup plus que par le passé les entendants, à prendre place dans la société, à mieux s'y insérer. Ce faisant, ils ont évolué et, dans le même temps qu'elle revendique son identité, la nouvelle génération souhaite être bilingue. Qui plus est, les sourds, grâce à l'interprétation qui s'est développée, ont désormais accès à bon nombre de manifestations culturelles. Cette confrontation à la culture ne suscite aucun rejet chez ceux qui n'ont pas pu poursuivre d'études. Bien au contraire, ils prennent conscience de ce qui leur a manqué et en éprouvent les plus vifs regrets.

D. S.: S'agissant de l'interprétation que vous avez évoquée à plusieurs reprises, quels sont les besoins des sourds?

A. M.: Avant que ne se développe l'interprétation de conférence en LSF, c'étaient essentiellement les enfants entendants de parents sourds qui faisaient office d'interprètes. Ils n'avaient reçu aucune formation. Les sourds faisaient appel à eux pour rencontrer, par exemple, un notaire ou un avocat, les échanges se déroulaient à huis clos et les sourds étaient satisfaits lorsqu'ils avaient bien compris l'interprète. Leur seul critère d'évaluation possible en la matière était le degré de maîtrise de la LSF de l'interprète. Celui-ci, très 
impliqué dans le monde de la surdité, était tout autant un conseiller qu'un interprète à proprement parler. À l'occasion de conférences, de réunions, la situation était tout à fait différente. Les entendants sont de plus en plus nombreux à apprendre la LSF et, sans être capables d'interpréter simultanément, ils comprennent suffisamment la LSF pour évaluer la fidélité de l'interprétation. En leur demandant leur avis à ce sujet, les sourds ont découvert que les interprétations de français en LSF ou de LSF en français laissaient à désirer parce qu'elles étaient approximatives, lacunaires ou reflétaient par trop l'opinion de l'interprète. En bref, dans l'ensemble, les interprétations n'étaient pas suffisamment fidèles.

Le fait que désormais les sourds prennent leur destin en main de plus en plus activement a entraîné chez eux une attente radicalement différente s'agissant de l'interprétation. Ils veulent désormais des interprètes de haut niveau très qualifiés qui doivent être à même de fournir des prestations équivalentes à celles des interprètes de conférences. Cela est capital pour qu'ils puissent assister à toutes les conférences, s'y exprimer, participer pleinement à toutes les réunions de travail, suivre les stages de formation, les cours, y compris à l'université. Les administrations et tous les services publics qui reçoivent non seulement des entendants, mais aussi des sourds, devraient faire un effort en matière d'interprétation. À ce propos, nous essayons d'obtenir des pouvoirs publics le droit pour les sourds de bénéficier annuellement d'un certain nombre, environ 25 , d'interprétations gratuites. Il faut que les sourds aient accès à la culture, à la formation, à l'information, qu'ils puissent exercer pleinement leur citoyenneté mais, bien évidemment, on l'aura compris, il ne s'agit pas de réclamer quotidiennement un interprète pour tous les actes de la vie courante.

L'interprétation permet aux sourds d'avoir accès in vivo à l'information. Autrefois, il y a une vingtaine d'années, en matière de politique les sourds étaient très influencés par leur entourage immédiat, souvent ils votaient comme leurs parents. Ils n'avaient pas accès aux débats télévisés à l'occasion desquels les candidats présentent leur programme. Désormais, des meetings sont interprétés, le débat opposant les deux candidats à la dernière élection présidentielle a été rediffusé accompagné d'une interprétation en LSF. Cela représente un gros progrès. Si on leur en donne les moyens, les sourds peuvent être des citoyens responsables et autonomes.

Je voudrais indiquer que nous souhaitons que, comme aux États-Unis ou en Suède, par exemple, les sourds français puissent bénéficier d'interprètes pour poursuivre leurs études, et ce, quel que soit le niveau considéré. Cela implique, il n'est pas inutile de le répéter, une formation de qualité et des candidats ayant une bonne culture générale. C'est pourquoi, il y a quelques années, notre fédération a soutenu la création d'une formation à l'ESIT. Il y a un point qui mérite d'être souligné et sur lequel on doit attirer l'attention des futurs interprètes. Lors de leurs prestations, les interprètes sont tenus de traduire fidèlement non seulement les entendants, mais aussi les sourds. Ils doivent s'interdire de parler à leur place, d'escamoter si peu que ce soit leurs discours, et ce, quelle qu'en soit la teneur. Autrement dit, ils doivent avoir la même attitude avec les sourds qu'avec les entendants.

D. S.: Vous n'avez évoqué que l'interprétation vers ou à partir de la LSF. Que pensezvous de la prise de notes en français?

A. M.: Le sous-titrage est le moyen qui s'impose pour les films mais, dès qu'il s'agit d'une réunion, d'une conférence ou d'un entretien, l'interprétation est préférable. Il est vrai que pour les sourds ou les devenus sourds qui ne pratiquent pas la LSF, la prise de notes des interventions diffusées à l'aide d'un rétroprojecteur est absolument nécessaire. Mais les sourds qui connaissent la LSF préfèrent l'interprétation. Elle n'introduit pas le décalage inhérent à la prise de notes, elle est plus vivante et elle permet une participation plus active, notamment aux réunions. 
D. S.: L'interprétation va aider à la promotion des sourds, leur permettre de poursuivre des études avec plus de chance de réussite, est-ce qu' indirectement elle ne va pas les aider à mieux maîtriser le français?

A. M. : Bien évidemment, oui. Les sourds, grâce à l'interprétation, comprennent mieux les cours, cela leur permet de lire plus aisément leurs manuels et dans le même temps d'enrichir leur français. Cela ne vaut pas seulement pour les étudiants. L'interprétation, en donnant accès au contenu, facilite la lecture des textes traitant des sujets abordés. Cela dit, j'aimerais attirer l'attention sur le fait que, contrairement à une idée reçue, le degré d'insertion et le niveau de culture générale des sourds ne dépendent pas uniquement de leur niveau de connaissance du français. Certains sourds n'ayant pas poursuivi de longues études, ayant une connaissance médiocre du français, peuvent se révéler des passionnés d'histoire, d'art, de peinture, de sculpture, des comédiens remarquables. Par ailleurs, ils peuvent, dans le cadre d'activités associatives ou d'actions à mener auprès des pouvoirs publics, faire preuve de réelles capacités d'organisation et de gestion. Enfin, tant dans leurs rapports avec les sourds qu'avec les entendants, ils peuvent tisser un réseau de relations, d'amis comme tout un chacun. A contrario, certains sourds - ils répondaient en cela à l'attente de leurs parents - pour avoir consacré dès leur plus tendre enfance toute leur énergie à l'acquisition du français parlé et écrit, se retrouvent bien démunis à l'âge adulte. Démunis en ce sens que l'étude de la langue a pris une telle place chez eux qu'elle a parfois laissé trop peu de place au développement de l'autonomie, de l'ouverture à autrui, de la curiosité intellectuelle, du sens artistique. L'épanouissement individuel est, au même titre que la maîtrise du français, un facteur déterminant d'intégration et de promotion sociale.

En fait, il s'agit de permettre aux sourds de s'épanouir harmonieusement. La langue des signes participe à cet épanouissement parce que les sourds l'acquièrent et la «parlent» tout aussi naturellement que les entendants s'agissant de la langue vocale. C'est pourquoi, dans le cadre d'un bilinguisme judicieusement mis en œuvre, elle devient un facteur facilitant l'acquisition du français chez le jeune et son approfondissement chez l'adulte.

Note

1. La Fédération Nationale des Sourds de France (FNSF) regroupe 62 associations. 